\title{
The Synthesis and Coordination Chemistry of a Photoswitchable Bis(phosphine) Ligand
}

\author{
David Sud, Robert McDonald ${ }^{\dagger}$ and Neil R. Branda* \\ Department of Chemistry, Simon Fraser University, 8888 University Drive, Burnaby, BC, \\ Canada V5A 1 S6
}

\section{SUPPORTING INFORMATION}

\section{Experimental}

General. All solvents used for synthesis were dried and degassed by passing them through steel columns containing activated alumina under nitrogen using an MBraun solvent purification system. All other solvents were used as received. Solvents for NMR analysis (Cambridge Isotope Laboratories) were used as received. Column chromatography was performed using silica gel 60 (230-400 mesh) from Silicycle Inc. All other reagents and starting materials were purchased from Aldrich. The 1,2-bis(5-chloro-2-methylthien-3-yl)hexafluorocyclopentene was prepared as described in literature. ${ }^{1}$

${ }^{1} \mathrm{H},{ }^{13} \mathrm{C}$ and ${ }^{31} \mathrm{P}$ NMR characterizations were performed on a Bruker AMX 400 instrument working at $400.10 \mathrm{MHz}$ for ${ }^{1} \mathrm{H} \mathrm{NMR}, 100.61 \mathrm{MHz}$ for ${ }^{13} \mathrm{C}$ NMR and $161.98 \mathrm{MHz}$ for ${ }^{31} \mathrm{P}$ NMR or a Varian Inova 500 instrument working at $499.77 \mathrm{MHz}$ for ${ }^{1} \mathrm{H}$ NMR. Chemical shifts $(\delta)$ are reported in parts per million relative to tetramethylsilane using the residual solvent peak as a reference standard. Coupling constants $(J)$ are reported in Hertz. FT-IR measurements were performed using a Nicolet Nexus 670 instrument. UV-vis measurements were performed using a Varian Cary 300 Bio spectrophotometer. X-ray diffraction studies were performed on a Bruker PLATFORM/SMART 1000CCD.

Photochemistry. The ring-closing reactions were carried out using the light source from a standard lamp used for visualizing TLC plates at $313 \mathrm{~nm}$ (Spectroline E-series, $470 \mathrm{~W} / \mathrm{cm}^{2}$ ). The ring-opening reactions were carried out using the light of a $150-\mathrm{W}$ tungsten source that was passed through a 434-nm cutoff filter to eliminate higher energy light.

\footnotetext{
${ }^{\dagger}$ X-Ray Crystallography Laboratory, Department of Chemistry, University of Alberta, Edmonton, AB, Canada T6G $2 \mathrm{G} 2$.

${ }^{1}$ Lucas, L. N.; van Esch, J.; Kellogg, R. M.; Feringa, B. L. Tetrahedron Lett. 1999, 40, 1775-1778.
} 
Synthetic scheme.

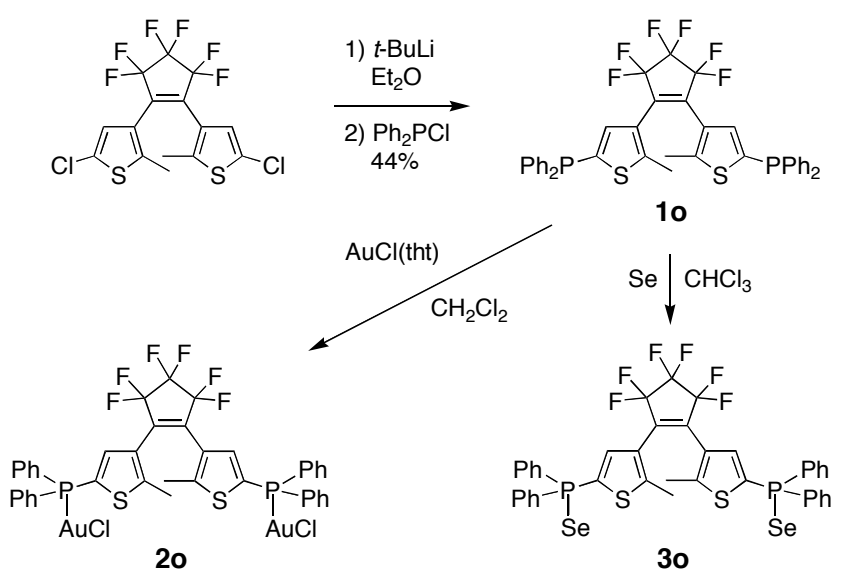

Synthesis of 1,2-bis(5-(diphenylphosphino)-2-methylthien-3-yl)hexafluorocyclopentene (10). Using a known procedure to prepare phosphinothiophene ligands, ${ }^{2}$ a solution 1,2-bis(5-chloro-2methylthien-3-yl)hexafluorocyclopentene $(250 \mathrm{mg}, 0.57 \mathrm{mmol})$ in anhydrous $\mathrm{Et}_{2} \mathrm{O}(50 \mathrm{~mL})$ cooled to $-78{ }^{\circ} \mathrm{C}$ was treated drop-wise with $t$-butyllithium $(0.94 \mathrm{~mL}, 1.7 \mathrm{M}$ in pentane, 1.60 mmol) under an $\mathrm{N}_{2}$ atmosphere. The reaction mixture was stirred at this temperature for $1 \mathrm{~h}$ at which time it was treated with $\mathrm{PPh}_{2} \mathrm{Cl}(226 \mu \mathrm{L}, 1.26 \mathrm{mmol})$ in one portion via a syringe. After the cold bath was removed and the reaction was slowly warmed to room temperature, it was stirred for $14 \mathrm{~h}$. The solution was concentrated to dryness in vacuo. The crude product was dissolved in $\mathrm{CH}_{2} \mathrm{Cl}_{2}(5 \mathrm{~mL})$ and was purified by passing it through a small silica plug using $\mathrm{CH}_{2} \mathrm{Cl}_{2}$ as the eluent. The product was further purified by flash chromatography through silica using $15 \% \mathrm{CH}_{2} \mathrm{Cl}_{2}$ in hexanes yielding $170 \mathrm{mg}$ (44\%) of 10 as white crystals after recrystallization from ethanol. M.p.154-157 ${ }^{\circ} \mathrm{C} .{ }^{1} \mathrm{H}$ NMR (499.77 MHz, $\left.\mathrm{CD}_{2} \mathrm{Cl}_{2}\right): \delta 7.40-7.30(\mathrm{~m}, 20 \mathrm{H}), 7.27(\mathrm{~d}, J=6 \mathrm{~Hz}, 2 \mathrm{H})$, $1.94(\mathrm{~s}, 6 \mathrm{H}) .{ }^{13} \mathrm{C}$ NMR $\left(100.61 \mathrm{MHz}, \mathrm{CD}_{2} \mathrm{Cl}_{2}\right): \delta 137.4(\mathrm{~d}, J=9 \mathrm{~Hz}), 136.3,136.0,133.0(\mathrm{~d}, J=$ $20 \mathrm{~Hz}$ ), 129.2, 128.7, 128.6, 14.6 (8 of 12 carbons found). ${ }^{31} \mathrm{P} \mathrm{NMR}\left(161.98 \mathrm{MHz}, \mathrm{CD}_{2} \mathrm{Cl}_{2}\right) \delta-$ 18.7 (s). FT-IR (KBr pellet) 3438, 3071, 3051, 2917, 2852, 1957, 1886, 1809, 1429, 1274, 1185 , 1114, 1088, 972, 747, 695, $496 \mathrm{~cm}^{-1}$. Anal. Calcd for $\mathrm{C}_{39} \mathrm{H}_{28} \mathrm{P}_{2} \mathrm{~F}_{6} \mathrm{~S}_{2}: \mathrm{C}, 63.58 ; \mathrm{H}, 3.83$. Found: C, 63.25; H, 3.61.

\section{Synthesis of 1,2-bis(5-((chlorogold)diphenylphosphino)-2-methylthien-3-}

yl)hexafluorocyclopentene 2o. Following previously reported preparation of gold complexes of phosphinothiophene ligands, ${ }^{3}$ a solution of $\mathrm{AuCl}(\mathrm{tht})(44 \mathrm{mg}, 0.14 \mathrm{mmol})$ in $\mathrm{CH}_{2} \mathrm{Cl}_{2}(10 \mathrm{~mL})$ was treated drop-wise with a solution of $10(50 \mathrm{mg}, 0.07 \mathrm{mmol})$ in $\mathrm{CH}_{2} \mathrm{Cl}_{2}(5 \mathrm{~mL})$ at $25{ }^{\circ} \mathrm{C}$. After stirring at this temperature for $14 \mathrm{~h}$, the solution was evaporated to dryness in vacuo. The product was purified by flash chromatography through silica using $25 \% \mathrm{CH}_{2} \mathrm{Cl}_{2}$ in hexanes yielding $15 \mathrm{mg}(18 \%)$ of air-stable crystals of 20 suitable for X-ray structural analysis after crystallization from hexanes and $\mathrm{CH}_{2} \mathrm{Cl}_{2}$.

\footnotetext{
${ }^{2}$ Field, J. S.; Haines, R. J.; Lakoba, E. I.; Sosabowski, M. H.; J. Chem. Soc., Perkin Trans. 1 2001, 3352.

${ }^{3}$ Clot, O.; Akahori, Y.; Moorlag, C.; Leznoff, D. B.; Wolf, M. O.; Batchelor, R. J.; Patrick, B. O.; Ishii, M.; Inorg. Chem., 2003, 42, 2704-2713.
} 
M.p. $217^{\circ} \mathrm{C}$ (dec.) ${ }^{1} \mathrm{H}$ NMR (499.77 MHz, $\left.\mathrm{CD}_{2} \mathrm{Cl}_{2}\right): \delta 7.64-7.48(\mathrm{~m}, 20 \mathrm{H}), 7.27(\mathrm{~d}, J=9 \mathrm{~Hz}$, 2H), 2.07 (s, 6H). ${ }^{31} \mathrm{P}$ NMR (161.98 MHz, $\left.\mathrm{CDCl}_{3}\right): \delta$ 19.8. FT-IR (KBr pellet) 1478, 1436, 1341, $1227,1204,1139,1101,986,746,692,537,524,502,491 \mathrm{~cm}^{-1}$.

\section{X-ray crystallography.}

1,2-bis(5-((chlorogold)diphenylphosphino)-2-methylthien-3-yl)hexafluorocyclopentene 20. Crystals of 20 were grown by slowly evaporating a solution of the photochrome in $50 \% \mathrm{CH}_{2} \mathrm{Cl}_{2}$ in hexanes. Crystal dimensions $(\mathrm{mm}) 0.47 \times 0.38 \times 0.33$, triclinic. The crystal structure was solved using direct methods (SHELXS-86) and refined by full-matrix least squares on $F^{2}$ (SHELXL-93).

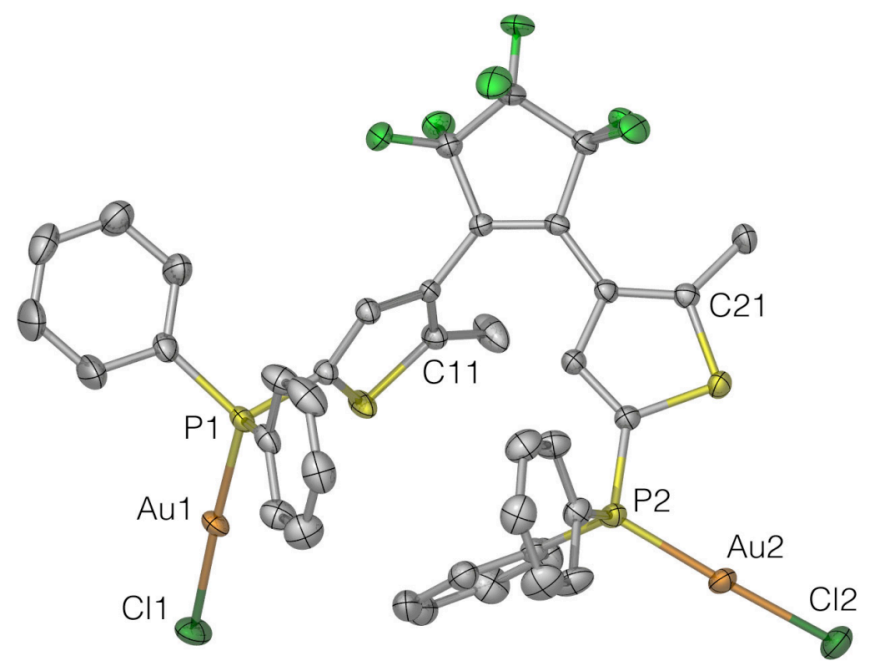

Figure S1. Molecular structure of 2o. Thermal ellipsoids are shown at 20\% probability. The hydrogen atoms are omitted for clarity.

Table S1. Crystallographic Data for 20.

\begin{tabular}{ll}
\hline Formula & $\mathrm{C}_{39} \mathrm{H}_{28} \mathrm{Au}_{2} \mathrm{Cl}_{2} \mathrm{~F}_{6} \mathrm{P}_{2} \mathrm{~S}_{2}$ \\
\hline Mol wt & 1201.51 \\
$T(\mathrm{~K})$ & 193 \\
Space group & $P 1(\mathrm{No.})$ \\
$a(\AA)$ & $9.7100(6)$ \\
$b(\AA)$ & $10.2317(6)$ \\
$C(\AA)$ & $20.4590(12)$ \\
$\alpha(\mathrm{deg})$ & $83.1693(9)$ \\
$\beta(\mathrm{deg})$ & $76.9049(8)$ \\
$\gamma(\mathrm{deg})$ & $84.1473(9)$ \\
$V\left(\AA^{3}\right)$ & $1959.8(2)$ \\
$\rho_{c}(\mathrm{~g} \mathrm{~cm}$ & $-3)$ \\
$Z$ & 2.036 \\
$\mu\left(\mathrm{mm}^{-1}\right)$ & 2 \\
$R_{1}\left[F_{0}^{2} \geq 2 \sigma\left(F_{0}^{2}\right)\right]$ & 7.860 \\
& 0.0214
\end{tabular}


$w R_{2}\left[F_{0}^{2} \geq-3 \sigma\left(F_{0}^{2}\right)\right]$

0.0542

Table S2. Selected Bond Lengths and Angles for 20.

\begin{tabular}{ll}
\hline & Distances, $\AA$ \\
\hline $\mathrm{Au}(1)-\mathrm{Au}(2)$ & $8.9954(4)$ \\
$\mathrm{Au}(1)-\mathrm{P}(1)$ & $2.2291(8)$ \\
$\mathrm{Au}(1)-\mathrm{Cl}(1)$ & $1.717(3)$ \\
$\mathrm{P}(1)-\mathrm{C}(14)$ & $1.795(3)$ \\
$\mathrm{P}(1)-\mathrm{C}(31)$ & $1.820(4)$ \\
$\mathrm{P}(1)-\mathrm{C}(41)$ & $1.818(4)$ \\
$\mathrm{C}(11)-\mathrm{C}(21)$ & $4.776(4)$ \\
& \\
$\mathrm{Cl}(1)-\mathrm{Au}(1)-\mathrm{P}(1)$ & Angles, deg \\
$\mathrm{Au}(1)-\mathrm{P}(1)-\mathrm{C}(14)$ & $177.68(3)$ \\
$\mathrm{Au}(1)-\mathrm{P}(1)-\mathrm{C}(31)$ & $109.56(11)$ \\
$\mathrm{Au}(1)-\mathrm{P}(1)-\mathrm{C}(41)$ & $113.87(12)$ \\
$\mathrm{P}(1)-\mathrm{C}(14)-\mathrm{S}(1)$ & $115.51(11)$ \\
$\mathrm{P}(1)-\mathrm{C}(14)-\mathrm{C}(13)$ & $118.66(18)$ \\
\hline
\end{tabular}

Synthesis of 1,2-bis(5-(diphenylphosphinoselenide)-2-methylthien-3-

yl)hexafluorocyclopentene 3o. Following a known procedure to prepare phosphine selenides, ${ }^{4,5}$ a solution of $10(50 \mathrm{mg}, 0.07 \mathrm{mmol})$ in $\mathrm{CHCl}_{3}(5 \mathrm{~mL})$ was treated with selenium $(43 \mathrm{mg}, 0.55$ $\mathrm{mmol}$ ) in one portion. The resulting solution was heated at reflux for $4 \mathrm{~h}$, when the heating source was removed and the reaction mixture was slowly cooled to room temperature. After stirring at this temperature for 30 minutes, the reaction mixture was filtered through a small silica plug using $\mathrm{CHCl}_{3}$ as the eluent yielding $60 \mathrm{mg}$ (98\%) of $\mathbf{3 o}$ as a yellowish solid.

M.p. 100-105 ${ }^{\circ} \mathrm{C} .{ }^{1} \mathrm{H}$ NMR (499.77 MHz, $\left.\mathrm{CDCl}_{3}\right): \delta 7.74-7.68(\mathrm{~m}, 8 \mathrm{H}), 7.56-7.48(\mathrm{~m}, 4 \mathrm{H}), 7.48-$ $7.42(\mathrm{~m}, 8 \mathrm{H}), 7.23(\mathrm{~d}, J=9 \mathrm{~Hz}, 2 \mathrm{H}), 2.02(\mathrm{~s}, 6 \mathrm{H}) .{ }^{13} \mathrm{C} \mathrm{NMR}\left(100.61 \mathrm{MHz}, \mathrm{CDCl}_{3}\right): \delta 136.8(\mathrm{~d}, J$ $=9 \mathrm{~Hz}), 133.8(\mathrm{~d}, J=83 \mathrm{~Hz}), 132.1(\mathrm{~d}, J=3 \mathrm{~Hz}), 132.0(\mathrm{~d}, J=11 \mathrm{~Hz}), 131.3,128.7(\mathrm{~d}, J=13$ $\mathrm{Hz}), 126.2$ (d, $J=13 \mathrm{~Hz}$ ), 14.7 (8 of 12 carbons found). ${ }^{31} \mathrm{P}$ NMR (161.98 MHz, $\mathrm{CDCl}_{3}$ ): $\delta 22.4$ (d, ${ }^{1} J^{77} \mathrm{Se}_{-}{ }^{31} \mathrm{P}=744 \mathrm{~Hz}$ ). Anal. Calcd. for $\mathrm{C}_{39} \mathrm{H}_{28} \mathrm{~S}_{2} \mathrm{~F}_{6} \mathrm{P}_{2} \mathrm{Se}_{2}$ : C, 52.36; H, 3.15. Found: C, 52.50; $\mathrm{H}, 3.37$.

Photochemical synthesis of ring-closed isomer 1c. A solution of 10 (1.60 mg, $2.17 \mathrm{mmol})$ in $\mathrm{CD}_{2} \mathrm{Cl}_{2}(0.5 \mathrm{~mL})$ was irradiated $(313 \mathrm{~nm})$ for 10 minutes yielding a solution containing $80 \%$ of 1c at the photostationary state as determined by ${ }^{1} \mathrm{H}$ NMR analysis. The remaining $20 \%$ was assigned to ring-open isomer $\mathbf{1 0 .}$

${ }^{1} \mathrm{H}$ NMR $\left(499.77 \mathrm{MHz}, \mathrm{CD}_{2} \mathrm{Cl}_{2}\right): \delta 7.52-7.37(\mathrm{~m}, 20 \mathrm{H}), 6.20(\mathrm{~d}, J=4 \mathrm{~Hz}, 2 \mathrm{H}), 2.06(\mathrm{~s}, 6 \mathrm{H}) .{ }^{31} \mathrm{P}$ $\operatorname{NMR}\left(161.98 \mathrm{MHz}, \mathrm{CD}_{2} \mathrm{Cl}_{2}\right) \delta-8.3(\mathrm{~s})$.

\footnotetext{
${ }^{4}$ Allen, D. W.; Taylor, B. F.; J. Chem. Soc., Dalton Trans., 1982, 51-54

${ }^{5}$ Chevykalova, M. N.; Manzhukova, L. F.; Artemova, N. V.; Luzikov, Y. N.; Nifant'ev, I. E., Nifant'ev, E. E.; Russ. Chem. Bull., 2003, 52, 78-84
} 
Photochemical synthesis of ring-closed isomer 2c. A solution of 20 (2.50 $\mathrm{mg}, 2.08 \mathrm{mmol})$ in $\mathrm{CD}_{2} \mathrm{Cl}_{2}(1.0 \mathrm{~mL})$ was irradiated $(313 \mathrm{~nm})$ for 10 minutes yielding a solution containing $60 \%$ of $\mathbf{2 c}$ at the photostationary state as determined by ${ }^{1} \mathrm{H}$ NMR analysis. The remaining $40 \%$ was assigned to ring-open isomer $\mathbf{2 0 .}$

${ }^{1} \mathrm{H}$ NMR (400.10 MHz, $\left.\mathrm{CD}_{2} \mathrm{Cl}_{2}\right): \delta 7.80-7.40(\mathrm{~m}, 20 \mathrm{H}), 6.45(\mathrm{~d}, J=10 \mathrm{~Hz}, 2 \mathrm{H}), 2.11(\mathrm{~s}, 6 \mathrm{H})$.

Photochemical synthesis of ring-closed isomer 3c. A solution of $\mathbf{3 o}(1.00 \mathrm{mg}, 1.11 \mathrm{mmol})$ in $\mathrm{CDCl}_{3}(0.5 \mathrm{~mL})$ was irradiated $(313 \mathrm{~nm})$ for 3 minutes yielding a solution containing $55 \%$ of 3c at the photostationary state as determined by ${ }^{1} \mathrm{H}$ NMR analysis. The remaining $45 \%$ was assigned to ring-open isomer 30.

${ }^{1} \mathrm{H}$ NMR (499.77 MHz, $\left.\mathrm{CDCl}_{3}\right): \delta 7.90-7.40(\mathrm{~m}, 20 \mathrm{H}), 6.64(\mathrm{~d}, J=11 \mathrm{~Hz}, 2 \mathrm{H}), 2.01(\mathrm{~s}, 6 \mathrm{H}) .{ }^{31} \mathrm{P}$ NMR (161.98 MHz, $\left.\mathrm{CD}_{2} \mathrm{Cl}_{2}\right) \delta 27.03\left(\mathrm{~d},{ }^{1} \mathrm{~J}^{77} \mathrm{Se}_{-}{ }^{31} \mathrm{P}=756 \mathrm{~Hz}\right)$.

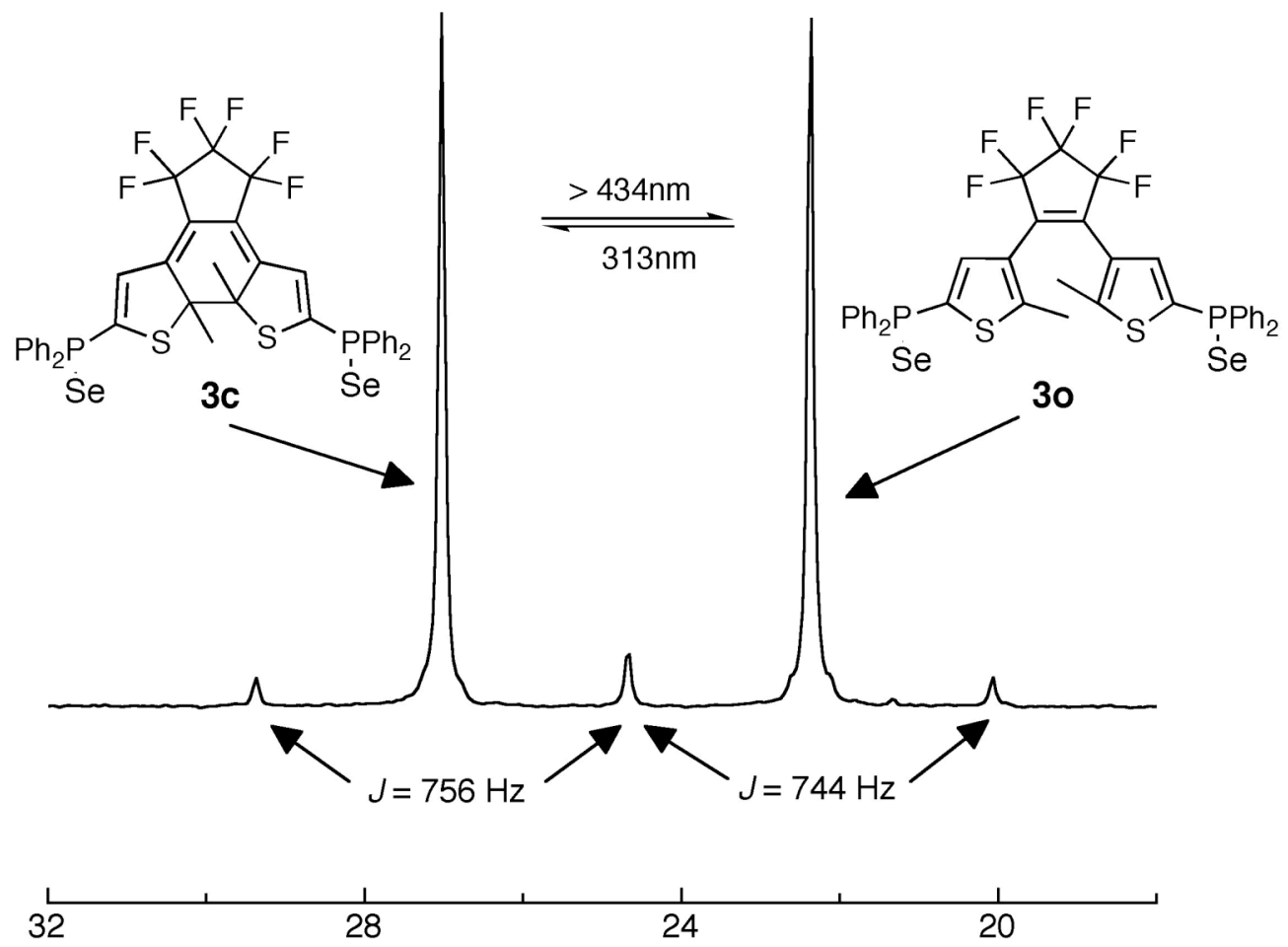

Figure S2. ${ }^{31} \mathrm{P}$ NMR spectrum of a mixture of the photostationary state generated irradiating a $\mathrm{CDCl}_{3}$ solution of 30 with $313-\mathrm{nm}$ light. The ${ }^{77} \mathrm{Se}_{-}{ }^{31} \mathrm{P}$ spin-spin coupling is highlighted. 